\title{
Condiciones de integración y fragmentación en la configuración de comunidades académicas en una universidad pública hondureña ${ }^{5}$
}

\section{Conditions of integration and fragmentation in the configuration of academic communities in a honduran public university}

Elma Barahona Henry*

ebarahona@upnfm.edu.hn

\section{Resumen}

La comunidad académica, es un concepto que surgió con la universidad moderna de investigación en Alemania (Clark, 1997), con el propósito de cultivar la ciencia a través de la investigación científica. En la actualidad, a toda institución que se denomine universidad, se le exige desarrollar investigación, sin embargo, esto será posible si cuentan con una comunidad académica, la cual puede encontrarse en diferentes grados de madurez en cuanto al desarrollo de la disciplina científica y la interdisciplina. Dado lo anterior, se presentan los resultados obtenidos al estudiar la comunidad académica en el contexto de una universidad pública con modelo profesionalizante en Honduras, a fin de comprender las condiciones de integración y fragmentación que influyen en su configuración. Los resultados evidenciaron dos macro dinámicas de interacción que se presentan a nivel institucional y que explican las condiciones de integracion y fragmentación en la configuracion de comundiades académicas, siendo estas: (a) conflicto y estacamiento conceptual versus fragmentación-rigidez estructural y (b) condiciones para el cambio progresivo a nivel conceptual y estructural. A partir de estas dinámicas, identificaron tres

${ }^{5}$ Este artículo está basado en los resultados obtenidos en la tesis doctoral "Configuración de comunidades académicas en la Universidad Nacional Autónoma de Honduras. El caso de Psicología, Medicina y Economía", para optar al grado de Doctor en Educación en el programa de Doctorado en Educación de la Universidad Pedagógica Nacional FM.

* Universidad Pedagógica Nacional Francisco Morazán.

Recibido 24 de mayo de 2017 /Aceptado 3 de junio de 2017. 
perspectivas para desarrollar futuras investigaciones, como ser: la perspectiva teorica-metodologica y etica, perspectiva desde la gestion y administracion universitaria, y la perspectiva desde gestión de la educación superior.
Palabras clave: Comunidad académica, integración, fragmentación, configuración, profesionalizante.

\section{Abstract}

The academic community is a concept that emerged with the modern research university in Germany (Clark, 1997), with the purpose of cultivating science through scientific research. At present, any institution called a university is required to develop research; however, this will be possible if they have an academic community which can be found in different degrees of maturity in the development of scientific discipline and interdiscipline. This is how the results obtained when studying the academic community are presented in the context of a public university with a professional model in Honduras, in order to understand the conditions of integration and fragmentation that influence its configuration.

Keywords: Academic community, integration, fragmentation, configuration, professionalizing.

\section{Introducción}

El estudio de la comunidad académica, ha sido abordado desde dos perspectivas: una "normativa" y otra "interpretativa" de la ciencia, considerándose como dos formas de análisis opuestos respecto al trabajo científico que se realiza en una comunidad. Desde la perspectiva normativa se discute fundamentalmente sobre el "ser y deber ser" de esta particular forma de agrupación basado en la disciplina científica y ethos de la ciencia (Becher, 2001, Merton,
2002, Kunh, 1971,1978). Desde la perspectiva interpretativa, el objetivo se centra en comprender cómo las normas que rigen el trabajo científico son interpretadas a lo interno de una comunidad y los factores externos que pueden influir en su comprensión y aplicación (Law y French, 1994, Mulkay, 1994), y por tanto distinguir la distancia entre la norma y la práctica (FagotLagueault, 2011). la Universidad Pedagógica Nacional Francisco Morazán, de Honduras, se comparten bajo términos de la Licencia Creative Commons: Se permite que otros puedan descargar las obras y compartirlas con otras personas, siempre y cuando se reconozca su autoría, pero no se pueden cambiar de ninguna manera ni se pueden utilizer comercialmente. 
En este trabajo se propuso la complementariedad de ambas perspectivas, ya que interesa, en primer lugar, estudiar la comunidad académica en el contexto de una universidad con un modelo de profesionalizante, distinto al modelo de universidad de investigación que dio origen al concepto, y en segundo lugar, comprender la separación entre la norma y la práctica en un contexto institucional específico. Para analizar esta distancia, la investigación se realiza en la Universidad Nacional Autónoma de Honduras (UNAH) y el análisis se centra en tres casos de estudio: Psicología, Medicina y Economía, obteniendo para cada uno de los casos configuraciones particulares, en Psicología se denominó "anclamiento conceptual y metodológico", en Medicina se designó como "conflictos epistémicos y estructurales" y en Economía se nombró "absorción acrítica de la teoría económica". Posteriormente, se comparan las configuraciones obtenidas en cada caso, y se integran en un tercer nivel de análisis con el objetivo de responder a la pregunta de investigación: ¿Cómo se estructuran las condiciones de fragmentación-integración que ha seguido la configuración de comunidades académicas en los tres casos de estudio, en el contexto de la Universidad Nacional Autónoma de Honduras? 


\section{Métodos y materiales}

El análisis de las condiciones de integración-fragmentación en la configuración de comunidades académicas en el contexto de la UNAH, se realizó como un estudio cualitativo, desde la perspectiva interpretativa (Krause, 1995) correspondiendo a un nivel de análisis micro-sociológico (Schwartz y Jacobs, 1984).

\section{Diseño}

El diseño se estructuró en base a la Teoría Fundamentada (Strauss y Corbin, 2002), en donde se abordaron los niveles de análisis: Para el análisis descriptiva y primera etapa del análisis, se realizó la codificación abierta, en donde se analizaron las categorías teóricas propuestas e identificaron las sub-categorías emergentes. Para el análisis inferencial, se realizó la codificación axial y en este nivel se analizaron las configuraciones que adoptaron los componentes estructurales en Psicología, Medicina y Economía. Posteriormente se desarrolló la codificación selectiva en donde integraron las configuraciones identificadas en los tres casos de estudio para responder a la pregunta de investigación respecto a la condiciones de integración de una comunidad académica en el contexto de una universidad profesionalizaste.

\section{Categorías de análisis}

A partir de la revisión teórica y empírica se identificaron cinco categorías teóricas, las cuales se constituyeron en componentes estructurales de una comunidad académica. Estas categorías fueron: (a) trabajo académico, (a) libertad académica, (c) ethos académico, (d) profesión académica y (e) cultura académica. Estas categorías se aplicaron a cada uno de los casos estudiados (Psicología, Medicina y Economía) con el objetivo de analizar la distancia entre la norma y la práctica. Inicialmente para cada caso, se analizaron las configuraciones que adoptaron las categorías teóricas, encontrándose en el caso de Psicología, una configuración que se denominó "anclamiento conceptual y metodológico", en el caso de Medicina la configuración se nombró "conflictos epistémicos y estructurales" y en Economía se llamó "absorción acrítica de la teoría economía". A partir de estas configuraciones se integró el siguiente nivel de análisis en donde se obtuvo la configuración "co-construcción de las condiciones de integración y fragmentación de comunidades académicas" la Universidad Pedagógica Nacional Francisco Morazán, de Honduras, se comparten bajo términos de la Licencia Creative Commons: Se permite que otros puedan descargar las obras y compartirlas con otras personas, siempre y
cuando se reconozca su autoría, pero no se pueden cambiar de ninguna manera ni se pueden utilizer comercialmente. 


\section{Participantes}

Los participantes en este estudio fueron 15 docentes de la UNAH, siendo 5 docentes pertenecientes a cada caso (Psicología, Medicina y Economía). Inicialmente se consideró la participación de docentes que solo hubieran tenido experiencia en investigación y docencia, sin embargo, se encontró que los docentes se dedicaban en distintos momentos a desarrollar diferentes funciones como ser: investigación, docencia, vinculación social y gestión-administración, lo que indicó que la investigación no es una función que se desarrolla de forma exclusiva. En cuanto al grado académico, 6 (seis) tenían grado de doctorado, 7 (siete) grado de maestría y dos docentes con grado de licenciatura. Respecto al género, 8 (ocho) fueron hombres y 7 (siete) mujeres. En cuanto a la edad, los participantes se ubicaron entre una edad mínima de 39 y edad máxima de 67 años. Respecto a los años de servicio, el docente con menos años fue de 3 (tres) años y el que el docente que contaba con más años de servicio fue de 33 (años). Sobre la categoría docente, 5 (cinco) tenían categoría de profesores auxiliares y 10 (diez) docentes titulares.

Con relación a la Universidad Nacional Autónoma de Honduras (UNAH), es la primera universidad pública del país, según la Constitución de la República de Honduras (La Gaceta, 1982), el la responsable de organizar, dirigir, desarrollar el sistema de educación superior hondureño. Es la universidad centenaria del país que desarrolla más campos de conocimientos y forma profesionales a nivel de carreras tecnológicas, grado y postgrado.

\section{Instrumentos de recolección de datos}

La recolección de datos se realizó a través de dos instrumentos: (a) entrevistas semi-estructuradas a los participantes, (b) matriz de análisis de documentos. En cuanto a la entrevista semi-estructurada, esta de diseño en base a los componentes estructurales que se constituyeron a su vez en categorías teóricas, y fue analizada por jueces expertos en el tema de universidad. Respecto a la matriz de análisis de documentos, se diseñó en base a las categorías teóricas, lo que permitió el contraste con el discurso de los docentes obtenidos en la entrevista. 


\section{Técnicas de análisis de datos}

El análisis de los datos se realizó en dos niveles: un descriptivo y otro relacional. A nivel descriptivo se analizaron las categorías teóricas y emergentes; a nivel relacional, específicamente en el análisis selectivo, se obtuvo un paradigma que explicó las condiciones de integración y fragmentación que se ha seguido la configuración de comunidades académicas en el contexto de estudio. El análisis anterior se contrastó con fuentes documentales (informes y estadísticas institucionales, nacionales e internacionales), que sustentaron la construcción de la teoría emergente de los datos.

\section{Discusión teórica}

La comunidad académica es un concepto que surge a principios de siglo XX en el contexto de la Universidad Alemana de investigación, y se entiende como aquel grupo que se dedica a al desarrollo de la ciencia a través de la investigación, enseñanza y estudio de la misma. Sin embargo, cuando se analiza la producción científica de las universidades en el contexto hondureño, se encuentra que se emplea el concepto de comunidad académica, sin considerar que el mismo surgió en un contexto distinto al que se está aplicando, ya que una institución que se denomine universidad no significa que esté integrada por comunidades académicas desde el sentido normativo, como puede ser el caso de la universidad de modelo más orientado a formar profesionales que el Estado y las sociedades requieren, y por tanto, su función principal es la docencia y no la investigación. Lo anterior se refleja en un estudio desarrollado por Salmi (2009), donde analizó universidades que siguen modelo de investigación, encontrando que aún dentro las mismas se pueden identificar diferencias en cuanto al desarrollo científico, producto de las condiciones de contexto en donde se inserta la institución universitaria. La discusión anterior es relevante ya que puede caerse en el error de comparar la producción científica de las universidades sin considerar el modelo que la misma sustenta, desconociendo los factores de contexto que condicionan el desarrollo de la ciencia e investigación científica.

Dado lo anterior, se considera relevante estudiar la comunidad académica en un contexto diferente al que le dio origen y comprender la configuración que adopta el mismo, y en este sentido, conocer y comprender las condiciones de integración y fragmentación que influyen la Universidad Pedagógica Nacional Francisco Morazán, de Honduras, se comparten bajo términos de la Licencia Creative Commons: Se permite que otros puedan descargar las obras y compartirlas con otras personas, siempre y
cuando se reconozca su autoría, pero no se pueden cambiar de ninguna manera ni se pueden utilizer comercialmente. 
en su desarrollo. Es así, que para esta investigación se desarrolla una propuesta analítica producto de la revisión teórica y empírica (figura 1), identificándose cinco componentes estructurales en una comunidad académica: (a) trabajo académico (Barnett, 2008; Becher, 2001; Boyer, 1999; Clark, 1997), (b) ethos académico (Albornoz, 2007; Dill, 2008; Merton, 2002), (c) libertad académica (Albornoz, 2009; Nelson, 2010; Soriano, 2008), (d) profesión académica (Altbach, 2003; Brunner, 2007; Clark, 1987; Greadiaga, 1999), (c) cultura académica (Clark, 1980; Mora, 2001; Sporn, 1996; Välimaa, 2008). Estos componentes estructurales responden una perspectiva normativa de la ciencia, que explica el ser y deber ser desde ésta forma particular de organización, y desde una perspectiva interpretativa ( Law y French, 1994; Mulkay, 1994) se analiza cómo los integrantes de las comunidades académicas desarrollan su comprensión sobre la ciencia a través de un "proceso de construcción intersubjetiva de la objetividad científica" (Fagot-Laguaut, 2011).

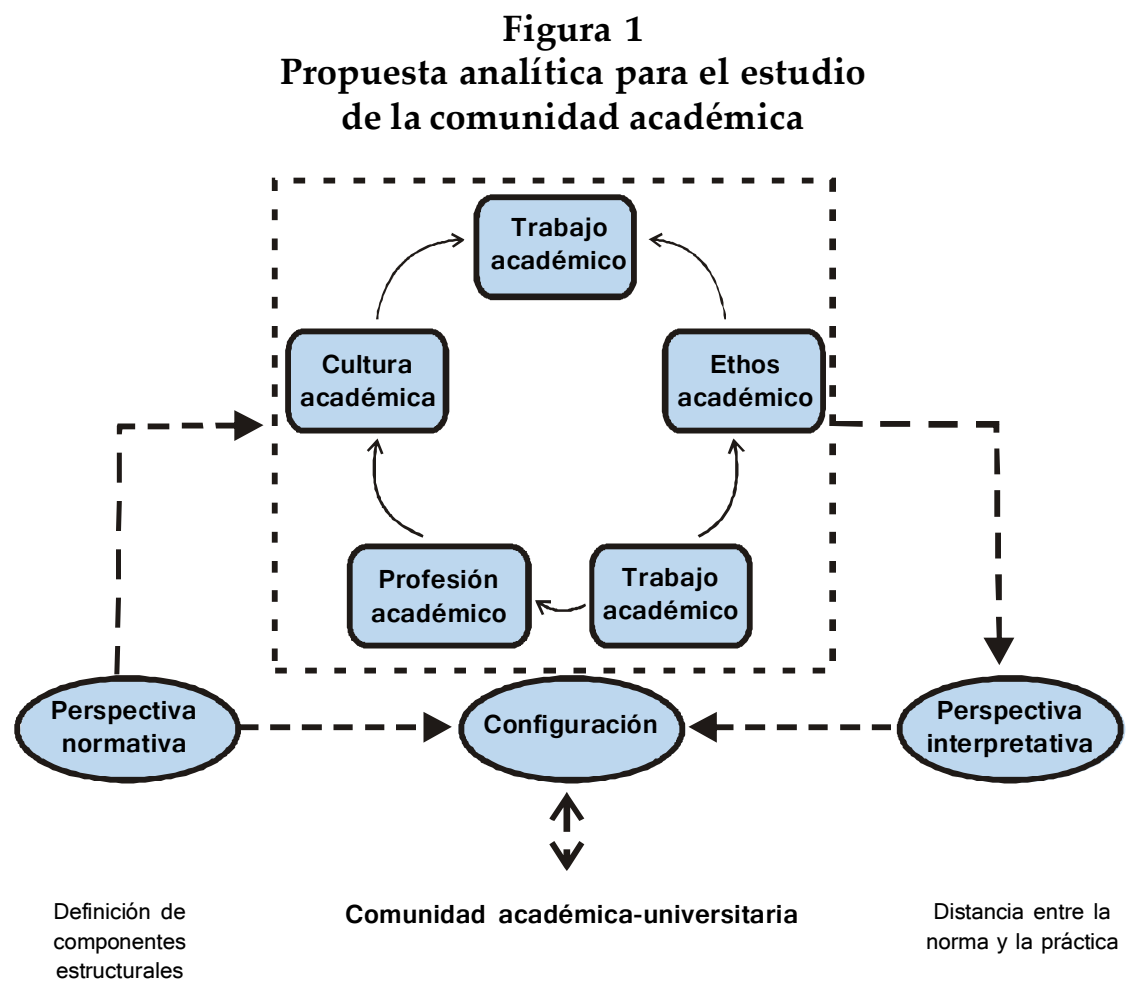

Fuente: Elaboración propia en base a las perspectivas normativa e interpretativa de la ciencia. 


\section{Análisis de resultados}

Los resultados sobre las condiciones de integración-fragmentación que ha seguido la configuración de las comunidades académicas en Psicología, Medicina y Economía en el contexto de la UNAH, indicaron una inter-relación entre ambas condiciones. A nivel de las condiciones contextuales-intervinientes, se produjo una dinámica que va de lo macro a lo micro y viceversa, y ésta se denominó (a) dinámica de conflicto y estancamiento conceptual versus fragmentación y rigidez contextual. Asimismo, se identificó otra dinámica denominada (b) condiciones para el cambio progresivo a nivel conceptual y estructural (figura 2).

\section{Figura 2}

\section{Condiciones de fragmentación-integración en la configuración de comunidades académicas en el contexto de la UNAH}

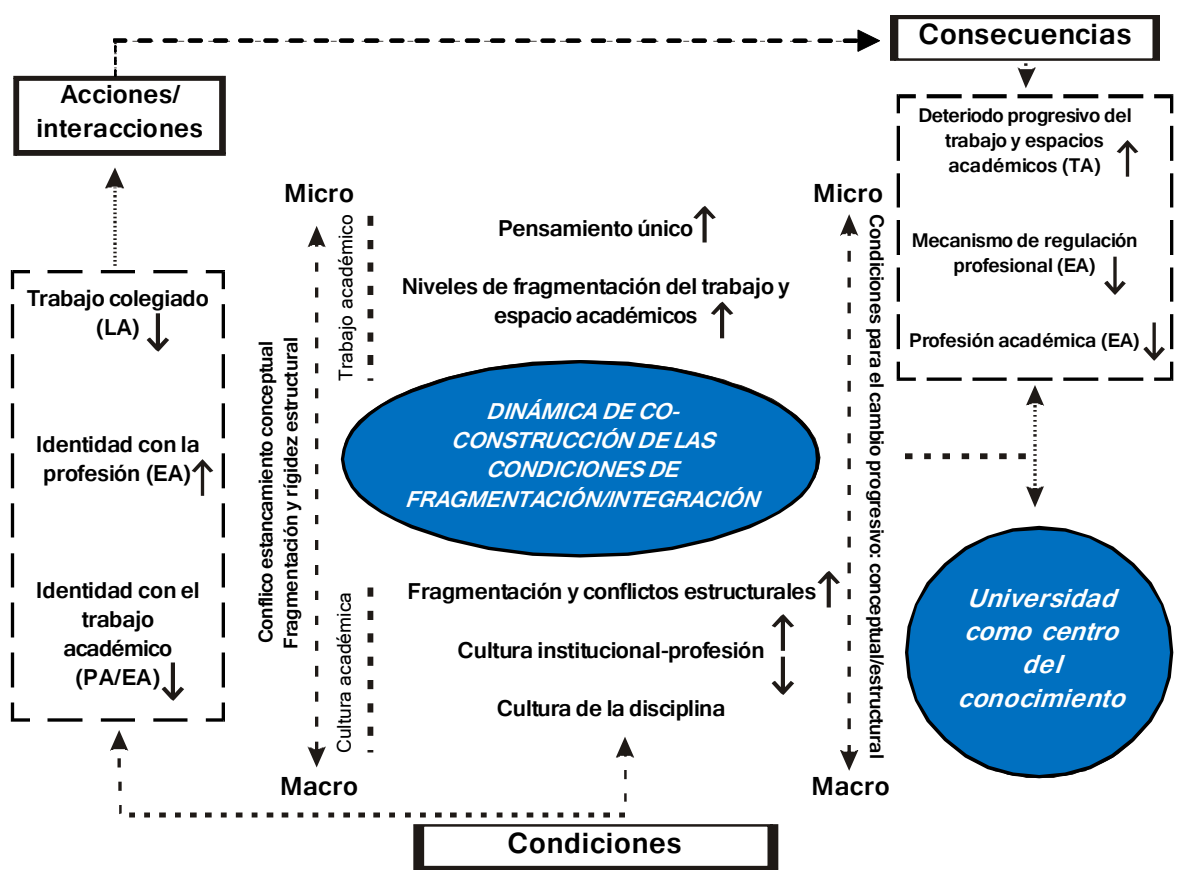

Fuente: Elaboración propia a partir de las configuraciones obtenidas en Psicología, Medicina y Economía. 
El conflicto y estancamiento conceptual/ fragmentación y rigidez contextual, se explicó a nivel macro, por la presencia de una cultura académica con niveles de (a) fragmentación y conflictos estructurales, (b) fuerte énfasis en la cultura de la institución y de la profesión y (c) una débil cultura académica. En relación a la fragmentación y conflictos estructurales, éste se relacionó como producto de estructuras de poder dominantes a nivel político y académico, las cuales se expresan a través vínculos entre los grupos más altos de la política académica-administrativa de la UNAH con sectores influyentes que están a lo externo de la universidad. Estas estructuras de poder forman parte de la cultura institucional denominada política universitaria las cuales tiene un fuerte peso en la definición de la vida institucional.

De igual forma, se encontró una separación entre el sector que trabaja el ámbito académico y el sector que trabaja lo administrativo, ambos sectores experimentaron en unos momentos históricos, relaciones de vinculación y en otros momentos, sus relaciones se concibieron como antagónicas, lo anterior debido a los propósitos que cada sector ha perseguido dentro de la institución. Es más, tratándose de una institución universitaria, la expectativa era que el sector administrativo respondiera a lo académico, situación que es explicada por Bourdieu (2008), desde el concepto de "campo". Un campo es un espacio estructurado conformado por posiciones y luchas, a través de las cuales se busca la preservación mediante disputas por el poder, las cuales se basan en identidad de sus miembros y en la jerarquía que ostentan dentro del campo y entre los campos (Wacquant, 2004). Es este caso, al estar presentes distintos campos dentro la universidad, las luchas entre ellos se explican por la preservación de uno en relación a otros.

Dentro de la cultura académica tambien se identificó un mayor enfasis en la cultura institucional y en la cultura de la profesión, y menos en la cultura disciplina cientifica la cual se considera la base para la conformación de las comunidades académcias. Esto se explicó por la fuerte presencia de una historia institucional compartida (Clark, 1980), centrada en la docencia y por tanto en la formacion de profesionales que la sociedad requiere. Sobre la cultura de la profesión se encontró que los docentes se identifican más como miembros de la profesión psicológica, médica y económica, y menos como miembros de una comundiad académica, debido su trabajo académico hace más énfasis en la comunicación de conocimieento a través de la docencia y menos en la creación de conocimiento a traves de la investigacion cientifica, lo que es reflejo de una débil cultura de la disciplina científica. 
A nivel micro se encontró en el componente estructural de trabajo académico la presencia de dos condiciones: (a) fuerte tendencia del pensamiento único, y (b) altos niveles de fragmentación en el trabajo y espacios académicos. El pensamiento único es la antítesis de lo que debe suceder en una institución universitaria, la cual debe caracterizarse por el cultivo del pensamiento múltiple (Albornoz, 2009; 2007; Bunge, 2001). Con esto no se plantea que estar en oposición es un discurso que por sí mismo refleja diversidad en el pensamiento, ya que puede ser la otra cara de la misma moneda y más estaría relacionado con discursos y prácticas de "populismo académico" (Albornoz, 2009).

El pensamiento diverso en una universidad se sustenta en lo epistemológico (Bunge, 2002; 2001; Soriano, 2008), y es a partir del mismo desde donde se construye la diversidad conceptual dentro de una institución universitaria. Los resultados en esta investigación, mostraron una fuerte tendencia al pensamiento único dentro de la universidad, que se produce entre lo macro y lo micro, ya que prácticamente se ha debilitado la construcción de un discurso epistemológicamente sólido y consistente que lleve según Albornoz (2007) a la construcción de marcos teóricos alternativos para interpretar la realidad. Por tanto, el conflicto y estancamiento en los marcos conceptuales refleja la dificultad de cambiar las "viejas estructuras conceptuales" y sustituirlas por "estructuras conceptuales innovadoras" lo que lleva a una fragmentación y rigidez estructural en el trabajo y espacios académicos. Por otro lado, la presencia de un fuerte pensamiento único, tienen como manifestaciones el "populismo académico" y la "mentalidad autoritaria" que se expresan en prácticas de "control y regulación del trabajo académico" (Albornoz, 2010; 2009; 2007).

Ante este conjunto de condiciones contextuales intervinientes, las estrategias de acción -interacción identificadas fueron: (a) un trabajo colegiado debilitado, (b) fuerte identidad referida al ejercicio de la profesión, y (c) la casi ausencia de un concepto de profesión académica. El debilitamiento del trabajo colegiado, se relacionó con la percepción desde los docentes, de la escasa importancia que tiene el trabajo académico. En los tres casos se pudo identificar a partir de los discursos, que la participación en reuniones de Departamento y de Facultad, estaba más centradas en los aspectos administrativos con fuerte peso dentro de la institución, diferente al trabajo colegiado que estaba más centrado en toma de decisiones cotidianas. En otros situaciones, cuando el trabajo académico se orientaba al desarrollo de tareas que por su baja la Universidad Pedagógica Nacional Francisco Morazán, de Honduras, se comparten bajo términos de la Licencia Creative Commons: Se permite que otros puedan descargar las obras y compartirlas con otras personas, siempre y cuando se reconozca su autoría, pero no se pueden cambiar de ninguna manera ni se pueden utilizer comercialmente. 
complejidad cognitiva, fueron percibidas como rutinarias, se identificaron conductas adaptativas relacionadas con mecanismos de "colegiación hueca" (Dill, 2008), en donde los profesores no están realmente involucrados en promover cambios e innovación en las actividades académicas. Se identificaron también, condiciones para el trabajo colegiado percibidas como satisfactorias, relacionadas con la toma de decisiones estrictamente académicas, sin embargo, no fueron la generalidad en los tres casos de estudio.

En relación con la identidad con la profesión, se encontró con docentes que se identificaban más con sus campos profesionales y menos con la profesión académica, esta última más propia de las instituciones universitarias que desarrollan fuertemente la investigación científica (Brunner, 2007; Grediaga Kuri, 1999). De ahí que aparezca en los discursos, la presencia de un rol profesional mayoritariamente definido por la profesión o a la figura del consultor. Es así como el estatus que pueden alcanzar como académicos no se produce por su trabajo dentro de la universidad, sino por su desempeño en otros escenarios laborales, de donde se obtiene mayor reconocimiento como expertos. En otros casos, en donde los docentes presentan un perfil más orientado hacia la profesión académica encuentra múltiples obstáculos, relacionadas con una estructura organizacional que mantienen prácticas y procesos organizacionales rígidos y poco innovadores para el desarrollo de la ciencia.

Los docentes consideraron como favorable la implementación un conjunto de políticas y normativas institucionales, así como diferentes mecanismos para impulsar la investigación científica, sin embargo, surgió el cuestionamiento sobre cómo las mismas afectarán el trabajo cotidiano, el cual está centrado en la docencia. También se identificaron obstáculos relacionados con estructuras externas, en el caso de Medicina, con el Sistema Nacional de Salud, ya que este no presenta las condiciones para la innovación científica e innovación, debido a una estructura nacional fragmentada y en algunos casos, ausente, y su consolidación se ve debilitada o interrumpida por los cambios en las políticas de los gobiernos de turno.

La discusión anterior permite delinear un conjunto de consecuencias como ser el (a) deterioro progresivo del trabajo y espacios académicos, (b) una disminución de los mecanismos de regulación profesional, y (c) un debilitamiento de la identidad con el trabajo académico. El deterioro 
progresivo de trabajo y espacios académicos, se consideró como el resultado de un conjunto de decisiones que orientaron la política institucional en determinados momentos históricos y que tuvieron un impacto negativo en el trabajo académico, ya que en vez de promover el desarrollo de nuevos marcos epistémicos que llevaran al desarrollo de nuevas prácticas académicas, se produjo un estancamiento tanto de marcos conceptuales como de prácticas relacionadas con una docencia de mayor calidad y el desarrollo de la investigación científica. Si bien, Medicina ha alcanzado niveles relativos de autonomía y libertad académica dada por las características de su campo, Psicología y Economía estuvieron permeadas por prácticas de ideologización y politización. Aunque, cuando los docentes analizaron las condiciones actuales en los campos, todos coincidieron en que el impacto fue negativo, siendo una de sus consecuencias el debilitamiento de los "mecanismos de regulación profesional" (Dill, 2008), producto de un deterioro del trabajo colegiado.

¿Cuáles son las perspectivas que se presentan para promover condiciones para el cambio conceptual/estructural? Se planteó la redefinición de la universidad como un centro de conocimiento, a través de aumentar el impacto social de rol que juega la universidad dentro de la sociedad, se considera urgente trabajar con estándares internacionales, así como potenciar la valoración de la ciencia e investigación científica. Esta propuesta es congruente con las dinámicas que se están produciendo en los escenarios internacionales de la Educación Superior, en donde se espera que las universidades aporten a los logros de los Objetivos del Milenio, a través de una redefinición de sus relaciones de colaboración como ser "Estado-empresa y tercer sector" (Maldonado, 2010), por otro lado, las universidades al estar insertas en lo que se denomina "sociedades del conocimiento" (UNESCO, 2005) o "economías del conocimiento" (Didriksson, 2008), implica que deben contar con comunidades académicas que avancen hacia altos "consensos cognitivos y normativos" (Law \& French, 1994), esto requeire según Fagot-Lagueault (2011), permanentemente construir su racionalidad, ya que las normas que rigen el trabajo académico pueden evolucionar o ser trasgedidas. la Universidad Pedagógica Nacional Francisco Morazán, de Honduras, se comparten bajo términos de la Licencia Creative Commons: Se permite que otros puedan descargar las obras y compartirlas con otras personas, siempre y
cuando se reconozca su autoría, pero no se pueden cambiar de ninguna manera ni se pueden utilizer comercialmente. 


\section{Conclusiones}

A partir de los resultados obtenidos se identifican dos dinámicas a estudiar e intervenir en diferentes niveles de la institucion: (a) conflictoestacamiento conceptual versus fragmentación-rigidez estructural; y (b) condiciones para el cambio progresivo a nivel conceptual y estructural. Lo anterior implica, que se deben retomar los enfoques sobre una universidad como institución ligada al conocimiento (Clark, 1997, entre otros) a fin de que la misma evolucione, considerándose características y grados de articulación presentes para cada nivel (macro-micro), y las posibilidades institucionales de consolidar progresivamente núcleos de trabajo que reflejen el modelo de comunidad académica desde lo normativo, hasta que paulatinamente se pueda entrar a un proceso sostenido de cambio institucional. Lo anterior es central, ya que la mayoría de los estudios sobre comunidades académicas se han realizado en universidades que siguen modelo de investigación (Becher, 2001; Salmi, 2009, entre otros), y ante el desafío de actual donde las instituciones universitarias deben desarrollar investigación científica, debe comprenderse cómo se configuran las comunidades académicas en universidades con modelo mas centrado en la docencia.

\section{Recomendaciones}

Con respecto a las implicaciones para la teoría y la práctica, a partir de los resultados se propuso trabajar desde las siguientes perspectivas: La perspectiva teórico-metodológica-ética indica que a nivel teórico, la investigación sobre comunidad académica, debe ser uno de los temas de agenda entre los investigadores y los tomadores de desiciones, ya que la dinámica de cambios que se están produciendo en la producción de conocimiento en escenarios nacionales, regionales e internacionales, tiene un impacto y en el rol que ésta institución pueda cumplir. A nivel metodológico, se propone estudiar este objeto de estudio con diferentes enfoques metodológicos, que integren diversidad de disciplinas científicas e instituciones unversitarias, a nivel nacional y regional. A nivel ético, el cuidado para quien estudia la comunidad académica, “(...) no es decir cómo deben comportarse, ni estigmatizar sus malas conductas. Lo que interesa es comprender el significado de la separación entre las normas y las prácticas" (Fagot-Lagueault, 2011, p. 150), ya que la racionalidad científica no es aprendida de forma pasiva, sino de forma constructiva en vinculación con el contexto (Mulkay, 1994).

<Paradigma> - Revista de Investigación Educativa. Año 24. No. 37 
Desde la perspectiva de la gestión-administración universitaria, los tomadores de decisiones al contar con información basada en resultados de investigación científica, pueden desarrollar nuevas formas de gestión y administración en las universidades, a fin de potenciarse a sí mismas como centros de producción de conocimiento, de ahí que, es fundamental impulsar investigación que les permita hacer lecturas más pertinentes del contexto interno y externo. A partir de la perspectiva de la gestión superior, debe considerarse que los problemas estructurales para el desarrollo de la ciencia y la investigación científica no son realidades de instituciones particulares, sino de cómo funcionan sistemas de Educación Superior. Alarcón (2013) sugirió que para la definición de políticas en este nivel, debe considerar los rasgos particulares y generales a nivel institucional, nacional, regional y en base a esto, tomar decisiones que impulsen la región en su conjunto. la Universidad Pedagógica Nacional Francisco Morazán, de Honduras, se comparten bajo términos de la Licencia Creative Commons: Se permite que otros puedan descargar las obras y compartirlas con otras personas, siempre y
cuando se reconozca su autoría, pero no se pueden cambiar de ninguna manera ni se pueden utilizer comercialmente. 


\section{Referencias Bibliográficas}

Alarcón, F. ( 2013). Panorámica de la Investigación y los Estudios de Postgrado en las Universidades de América Central y República Dominicana. Ponencia presentada en la Sesión Extraordinaria del CSUCA. Managua, Nicaragua: CSUCA.

Albornoz, O. (2009). Breves Notas sobre Autonomía y Libertad Académica. Caracas, Venezuela: EBUC. Albornoz, O. (2007). La Libertad Académica y la Mentalidad Autoritaria. Caracas, Venezuela: EBUC.

Albornoz, O. (2010). Regulación y Control Académico. Caracas, Venezuela: EBUC.

Altbach, P. (2003). Centers and peripheries in the academic profession: The special challenges of development countries. En P. Albaach, (Eds.), The Decline of Guru (pp. 1-23). EUA: Palgrave-MacMillan.

Barnett, R. (2008). Para una Transformación de la Universidad. Barcelona, España: Octaedro.

Becher, T. (2001). Tribus y Territorios Académicos. Barcelona, España: Gedisa.

Boyer, E. (2003). Una Propuesta para la Educación Superior del Futuro. México, D.F., México: Fondo de Cultura Económica.

Bunge, M. (2001). La Relación entre la Sociología y la Filosofía. Madrid, España: EDAF.

Brunner, J. (2009). Apuntes de sociología de la educación superior, en el contexto internacional, nacional y local. Estudios Pedagógicos, XXXV (2), 203-230. Extraído de http://mingaonline.uach.cl/pdf/estped/v35n2/ art12.pdf

Clark, B. (1997). Las Universidades Modernas como Espacios de Investigación y Docencia. México, D.F., México: Miguel Angel Porrúa. Press.

Clark, B. (1980). Academic Culture. New Haven, EUA: Yale University 
Didriksson, A. (2008). Contexto global y regional de la educación superior en América Latina. En A. G. (Eds), Tendencias de la Educación Superior en América Latina (pp. 23-53). Caracas, Venezuela: IESALCUNESCO.

Dill, D. (2008). La degradación de la ética académica: Docencia, investigación y renovación de la autorregulación profesional. En R. Barnett, Para una Transformación de la Universidad (pp. 231-246). Madrid, España: Octaedro.

Fagot-Lagueault, A. (2011). La construcción intersujetiva del la objetividad científica. En A. Andler, A. Fagot-Laguealt, y B. Saint-Sernin (Editores), Filosofía de las Ciencias. México, D.F., México: Fondo de Cultura Económica.

Grediaga Kuri, R. (1999). Profesión Académica, Disciplinas y Organizaciones. Tesis de Doctorado en Sociología. El Colegio de México. México, D.F., México: ANUIES.

Kuhn, T. (1971). La Estructura de las Revoluciones Científicas. México, D.F, México: Fondo de Cultura Económica.

Kuhn, T. (1978). Segundos Pensamientos sobre Paradigmas. Madrid, España: Tecnos.

Krause, M. (1995). La investigación cualitativa: Un campo de posibilidades y desafíos. Revista Temas de Educación, 7, 19-39.

La Gaceta. (20 de enero de 1982). Constitución de la República de Honduras. Diario Oficial de la República de Honduras.

Law, J. y French, D. (1994). Sociologías normativa e interpretativa de la ciencia. En J. Iranzo, J. Blanco, T. Gonzáles de la Fe, C. Torres, y A. Cotillo (Editores), Sociología de la Ciencia y la Tecnología (pp. 53-63). Madrid, España: CSIC.

Merton, R. (2002). Teoría y Estructuras Sociales. México, D.F, México: Fondo de Cultura Económica.

Mulkay, M. (1994). La visión sociológica habitual de la ciencia. En J. Iranzo, T. González de la Fe, C. Torres, \& A. Cotillo (Coord.), Sociología 
de la Ciencia y la Tecnología (pp. 13-32). Madrid, España: CSIC.

Mora, J.-G. (1994). Governance and management in the new university. Tertiary Education and Management (7), 95-100. DOI: 10.1023/ A:101338016085.

Nelson, G. (2010). No University is an Island: Saving Academic Freedom. NY, EUA: NY Academic Press.

Salmi, J. (2009). El Desafio de Crear Universidades de Rango Mundial. Washington, EUA: Banco Mundial.

Schwartz, J.\& Jacobs, J. (1984). Sociología Cualitativa. México, D.F. México: Trillas.

Soriano, O. (2008). Los estudios de postgrado: Libertad académica y líneas de investigación. En O. Soriano, (Coord.), Problemas Educativos en la Sociedad Contemporánea (pp. 9-28). Tegucigalpa, Honduras: UPNFM.

Sporn, B. (1996). Managing university culture: An analysis of the relation between institutional culture and management approaches. Higher Education (32), 41-61. DOI:10.1007/BF00139217

Strauss, A.\& Corbin, J. (2002). Bases de la Investigación Cualitativa. Técnicas y Procedimientos para el Desarrollo de la Teoría Fundamentada.Bogotá, Colombia: Universidad de Antioquia.

UNESCO. (2005). Informe Mundial: Hacia las Sociedades del Conocimiento. Jouve, Francia: UNESCO.

Välimaa, J. (2008). Cultural studies in higher research. En J. Välimaa, (Eds.), Cultural Perspective on Higher Education (pp. 9-25). Finland: Springer. 